$\mathbf{T}_{\text {he }} \mathbf{J}_{\text {ournal of }} \mathbf{N}_{\text {onlinear }} \mathbf{S}_{\text {ciences and }}$ Applications

http://www.tjnsa.com

\title{
ON MULTIPOINT ITERATIVE PROCESSES OF EFFICIENCY INDEX HIGHER THAN NEWTON'S METHOD
}

\author{
IOANNIS K. ARGYROS ${ }^{1 *}$ AND SAÏD HILOUT ${ }^{2}$
}

\begin{abstract}
We provided a convergence analysis of third-order multipoint iterative processes of efficiency index higher than Newton's.

Our convergence analysis is finer than the corresponding one in [8, under the same or weaker hypotheses and computational cost.
\end{abstract}

\section{INTRODUCTION}

In this study we are concerned with the problem of approximating a locally unique solution $x^{\star}$ of equation

$$
F(x)=0,
$$

where $F$ is a Fréchet-differentiable operator defined on a non-empty open, and convex $\mathcal{D}$ of a Banach space $\mathcal{X}$ with values in a Banach space $\mathcal{Y}$.

A large number of problems in applied mathematics and also in engineering are solved by finding the solutions of certain equations. For example, dynamic systems are mathematically modeled by difference or differential equations, and their solutions usually represent the states of the systems. For the sake of simplicity, assume that a time-invariant system is driven by the equation $\dot{x}=Q(x)$, for some suitable operator $Q$, where $x$ is the state. Then the equilibrium states are determined by solving equation (1.1). Similar equations are used in the case of discrete systems. The unknowns of engineering equations can be functions (difference, differential, and integral equations), vectors (systems of linear or nonlinear algebraic equations), or real or complex numbers (single algebraic equations with

Date: Received: 2 March 2009; Revised: 15 June 2009.

* Corresponding author.

2000 Mathematics Subject Classification. Primary 65H10, 65H05, 65H10, 47H99, 49M15.

Key words and phrases. Banach space, Multipoint iterative procedure, Fréchet-derivatives, Newton-type method, Majorizing sequences. 
single unknowns). Except in special cases, the most commonly used solution methods are iterative--when starting from one or several initial approximations a sequence is constructed that converges to a solution of the equation. Iteration methods are also applied for solving optimization problems. In such cases, the iteration sequences converge to an optimal solution of the problem at hand. Since all of these methods have the same recursive structure, they can be introduced and discussed in a general framework.

Motivated by optimization considerations, and the elegant work by Ezquerro and Hernández in [8], we study the Newton-Chebyshev-type method (NCTM), defined as follows:

$$
\begin{aligned}
& x_{0} \in \mathcal{D}, \\
& y_{n}=x_{n}-\Gamma_{n} F\left(x_{n}\right) \\
& z_{n}=x_{n}+p\left(y_{n}-x_{n}\right), \quad p \in(0,1], \\
& x_{n+1}=x_{n}-\frac{1}{p^{2}} \Gamma_{n}\left(\left(p^{2}+p-1\right) F\left(x_{n}\right)+F\left(z_{n}\right)\right), \quad n \geq 0,
\end{aligned}
$$

where, $\Gamma_{n}=F^{\prime}\left(x_{n}\right)^{-1},(n \geq 0)$.

Newton-type methods have been studied under various assumptions [1]-[10]. A convergence analysis was provided for (NCTM) under conditions similar to Newton's method. Note that (NCTM) Reduces to Newton's method, if $p=1$.

The derivation of (NCTM) as well as the justification for generating iterative procedures of better effeciency index than Newton's can be found in [8].

Here, we show how to provide a finer convergence analysis under the same or weaker hypotheses than in [8].

\section{Semilocal convergence analysis for (NCTM)}

Let $\ell_{0}>0, \ell>0$, and $\eta \geq 0$ be given constants. Scalar sequences $\left\{t_{n}\right\},\left\{s_{n}\right\}$ given by:

$$
\begin{gathered}
t_{0}=0, \quad s_{0}=\eta, \quad t_{1}=s_{0}+\frac{\ell_{0}}{2}\left(s_{0}-t_{0}\right)^{2}, \\
t_{n+1}=s_{n}+\frac{\ell\left(s_{n}-t_{n}\right)^{2}}{2\left(1-\ell_{0} t_{n}\right)} \quad(n \geq 2), \\
s_{1}=t_{1}+\frac{\ell_{0}\left(\left(s_{0}-t_{0}\right)^{2}+\left(t_{1}-t_{0}\right)^{2}\right)}{2\left(1-\ell_{0} t_{1}\right)}, \quad(n \geq 2), \\
s_{n}=t_{n}+\frac{\ell\left(\left(s_{n-1}-t_{n-1}\right)^{2}+\left(t_{n}-t_{n-1}\right)^{2}\right)}{2\left(1-\ell_{0} t_{n}\right)}, \quad
\end{gathered}
$$

shall be shown to be majorizing for $\left\{x_{n}\right\},\left\{y_{n}\right\}$. 
Note that if

$$
\ell_{0} t_{n}<1 \quad(n \geq 1)
$$

then, we obtain:

$$
s_{n-1} \leq t_{n} \leq s_{n} \leq t_{n+1}<\frac{1}{\ell_{0}} \quad(n \geq 1) .
$$

Sufficient conditions implying (2.5) can be found in [3], [6] (see, e.g. (2.38) for such a condition). Hence, we arrived at:

Lemma 2.1. Under condition (2.5), scalar sequences $\left\{t_{n}\right\},\left\{s_{n}\right\}$ are non-decreasing, bounded above by $r$ given by:

$$
r=\frac{1}{\ell_{0}}
$$

and converge to their unique least upper bound $r_{0}$ satisfying

$$
r_{0} \in[0, r] .
$$

We shall show the main semilocal convergence result for (NCTM).

Theorem 2.2. Let $F: \mathcal{D} \subseteq \mathcal{X} \longrightarrow \mathcal{Y}$ be a Fréchet-differentiable operator defined on a non-empty, open, and convex domain $\mathcal{D}$ of a Banach space $\mathcal{X}$ with values in a Banach space $\mathcal{Y}$.

Assume:

there exist $x_{0} \in \mathcal{D}$, constants $\ell_{0}>0, \ell>0$, and $\eta \geq 0$, such that for all $x, y \in \mathcal{D}$ :

$$
\begin{gathered}
\Gamma_{0} \in \mathcal{L}(\mathcal{Y}, \mathcal{X}), \\
\left\|\Gamma_{0} F\left(x_{0}\right)\right\| \leq \eta, \\
\left\|\Gamma_{0}\left(F^{\prime}(x)-F^{\prime}\left(x_{0}\right)\right)\right\| \leq \ell_{0} \quad\left\|x-x_{0}\right\|, \\
\left\|\Gamma_{0}\left(F^{\prime}(x)-F^{\prime}(y)\right)\right\| \leq \ell \quad\|x-y\|, \\
\bar{U}\left(x_{0}, r_{0}\right)=\left\{x \in \mathcal{X}:\left\|x-x_{0}\right\| \leq r_{0}\right\} \subseteq \mathcal{D},
\end{gathered}
$$

hypotheses of Lemma 2.1 hold, and

$$
\ell_{0} r_{0}<1
$$

Then,

(a) sequences $\left\{x_{n}\right\},\left\{y_{n}\right\},(n \geq 0)$ generated by (NCTM) are well defined, remain in $\bar{U}\left(x_{0}, r_{0}\right)$ for all $n \geq 0$, and converge to a solution $x^{\star} \in \bar{U}\left(x_{0}, r_{0}\right)$ of equation $F(x)=0$. 
The solution $x^{\star}$ is unique in

$$
U\left(x_{0}, \frac{2}{\ell_{0}}-r_{0}\right) \cap \mathcal{D}
$$

if

$$
r_{0}<\frac{2}{\ell_{0}}
$$

Moreover the following estimates hold for all $n \geq 0$ :

$$
\left\|x_{n}-x^{\star}\right\| \leq r_{0}-t_{n},
$$

and

$$
\left\|y_{n}-x^{\star}\right\| \leq r_{0}-s_{n} .
$$

(b) Assume $F$ is sufficiently differentiable in $\mathcal{D}$. If $F$ has a simple solution $x^{\star} \in \mathcal{D}, F^{\prime}(x)^{-1}$ exists in a neighborhood of $x^{\star}, x_{0}$ is sufficiently close to $x^{\star}, p \in(0,1]$, then (NCTM) has order of convergence at least three.

Proof. We shall show using induction on $m$ :

$$
\left\|y_{m}-x_{m}\right\| \leq s_{m}-t_{m} .
$$

and

$$
\left\|x_{m+1}-y_{m}\right\| \leq t_{m+1}-s_{m}
$$

Let $m=0$, then $(2.19)$ is true, since by $(2.1),(\mathrm{NCTM})$, and $(2.10)$ :

$$
\left\|\Gamma_{0} F\left(x_{0}\right)\right\| \leq \eta=s_{0}-t_{0}<r_{0}
$$

which also implies $y_{0} \in \bar{U}\left(x_{0}, r_{0}\right)$.

Let $x \in \bar{U}\left(x_{0}, r_{0}\right)$. Then, in view of 2.5), and 2.11, we have:

$$
\left\|\Gamma_{0}\left(F^{\prime}(x)-F^{\prime}\left(x_{0}\right)\right)\right\| \leq \ell_{0} \quad\left\|x-x_{0}\right\| \leq \ell_{0} r_{0}<1 .
$$

It follows from (2.21), and the Banach lemma on invertible operators [6], [7], [9] that $F^{\prime}(x)^{-1} \in \mathcal{L}(\mathcal{Y}, \mathcal{X})$, and

$$
\left\|F^{\prime}(x)^{-1} F^{\prime}\left(x_{0}\right)\right\| \leq \frac{1}{1-\ell_{0}\left\|x-x_{0}\right\|} \leq \frac{1}{1-\ell_{0} r_{0}} .
$$

Using Taylor's formula, and assuming $x_{m-1}, y_{m-1}, x_{m}, y_{m}$ exist, we have:

$$
\begin{aligned}
F\left(z_{m}\right)=(1-p) & F\left(x_{m}\right)+p \int_{0}^{1}\left(F^{\prime}\left(x_{m}+p \theta\left(y_{m}-x_{m}\right)\right)-F^{\prime}\left(x_{m}\right)\right)\left(y_{m}-x_{m}\right) d \theta, \\
x_{m+1}-y_{m} & =x_{m}-\frac{\Gamma_{m}}{p^{2}}\left(\left(p^{2}+p-1\right) F\left(x_{m}\right)+F\left(z_{m}\right)\right)-x_{m}+\Gamma_{m} F\left(x_{m}\right) \\
& =-\frac{1}{p^{2}} \Gamma_{m} F\left(x_{m}\right)+\left(1-\frac{p^{2}+p-1}{p^{2}}\right) \Gamma_{m} F\left(x_{m}\right) \\
& =-\frac{1}{p^{2}}\left(\Gamma_{m} F\left(z_{m}\right)-(1-p) \Gamma_{m} F\left(x_{m}\right)\right) .
\end{aligned}
$$


Then, by (2.23), 2.24) becomes:

$$
\begin{aligned}
x_{m+1}-y_{m}= & -\frac{1}{p^{2}} \Gamma_{m}\left((1-p) F\left(x_{m}\right)+p \int_{0}^{1}\left(F^{\prime}\left(x_{m}+\theta p\left(y_{m}-x_{m}\right)\right)-\right.\right. \\
& \left.\left.F^{\prime}\left(x_{m}\right)\right)\left(y_{m}-x_{m}\right) d \theta\right)+\frac{1-p}{p^{2}} \Gamma_{m} F\left(x_{m}\right) \\
= & -\frac{1}{p} \Gamma_{m} \int_{0}^{1}\left(F^{\prime}\left(x_{m}+\theta p\left(y_{m}-x_{m}\right)\right)-F^{\prime}\left(x_{m}\right)\right)\left(y_{m}-x_{m}\right) d \theta .
\end{aligned}
$$

We also have:

$$
\begin{aligned}
F\left(x_{m}\right)= & \frac{1}{p} \int_{0}^{1}\left(F^{\prime}\left(x_{m-1}\right)-F^{\prime}\left(x_{m-1}+p \theta\left(y_{m-1}-x_{m-1}\right)\right)\right)\left(y_{m-1}-x_{m-1}\right) d \theta+ \\
& \int_{0}^{1}\left(F^{\prime}\left(x_{m-1}+\theta\left(x_{m}-x_{m-1}\right)\right)-F^{\prime}\left(x_{m-1}\right)\right)\left(x_{m}-x_{m-1}\right) d \theta .
\end{aligned}
$$

We have $\left\|z_{0}-x_{0}\right\| \leq p \eta \leq \eta \leq r_{0}$, which imply $z_{0} \in \bar{U}\left(x_{0}, r_{0}\right)$. By hypothesis (2.9), $\Gamma_{0}$ exists. Hence, from $m=0$ in (2.25), $x_{1}$ is well defined. We shall show $x_{1} \in \bar{U}\left(x_{0}, r_{0}\right)$.

Indeed, using (2.1), 2.10), and 2.25), we get

$$
\left\|x_{1}-y_{0}\right\| \leq \frac{\ell_{0}}{2}\left\|y_{0}-x_{0}\right\|^{2} \leq \frac{\ell_{0}}{2}\left(s_{0}-t_{0}\right)^{2}=t_{1}-s_{0} \leq t_{1}-t_{0} \leq r_{0}
$$

which shows 2.20 for $m=0$, and $x_{1} \in \bar{U}\left(x_{0}, r_{0}\right)$.

It follows that $\Gamma_{1}, y_{1}$, and $z_{1}$ exist.

Using (NCTM), 2.2), 2.24 -2.26 for $m=1$, and setting $x=x_{1}$ in 2.22, we get:

$$
\begin{aligned}
\left\|y_{1}-x_{1}\right\| & \leq \frac{\ell_{0}\left(\left\|y_{0}-x_{0}\right\|^{2}+\left\|x_{1}-x_{0}\right\|^{2}\right)}{2\left(1-\ell_{0}\left\|x_{1}-x_{0}\right\|\right)} \\
& \leq \frac{\ell_{0}\left(\left(s_{0}-t_{0}\right)^{2}+\left(t_{1}-t_{0}\right)^{2}\right)}{2\left(1-\ell_{0} t_{1}\right)}=s_{1}-t_{1}
\end{aligned}
$$

and

$$
\begin{aligned}
\left\|x_{2}-y_{1}\right\| & \leq \frac{\ell}{2} \frac{\left\|y_{1}-x_{1}\right\|^{2}}{1-\ell_{0}\left\|x_{1}-x_{0}\right\|} \\
& \leq \frac{\ell}{2} \frac{\left(s_{1}-t_{1}\right)^{2}}{1-\ell_{0} t_{1}}=t_{2}-s_{1}
\end{aligned}
$$

which show 2.19 , and 2.20 for $m=1$.

We also have:

$$
\begin{aligned}
\left\|x_{2}-x_{0}\right\| & \leq\left\|x_{2}-y_{1}\right\|+\left\|y_{1}-x_{1}\right\|+\left\|x_{1}-x_{0}\right\| \\
& \leq t_{2}-s_{1}+s_{1}-t_{1}+t_{1}-t_{0}=t_{2} \leq r_{0},
\end{aligned}
$$

which implies $x_{2} \in \bar{U}\left(x_{0}, r_{0}\right)$. 
Hence, $\Gamma_{2}$ exists, and from an analogous procedure $y_{2}, z_{2}, x_{3} \in \bar{U}\left(x_{0}, r_{0}\right)$. Assume 2.19, 2.20, $x_{k}, y_{k}, x_{k+1} \in \bar{U}\left(x_{0}, r_{0}\right)$ hold for all $k \leq m$.

Then, using (NCTM), 2.2), 2.24)-(2.26), and setting $x=x_{m}$ in 2.22), we obtain as in (2.27), 2.28, respectively,

$$
\begin{aligned}
\left\|y_{m}-x_{m}\right\| & \leq \frac{\ell\left(\left\|y_{m}-x_{m}\right\|^{2}+\left\|x_{m}-x_{m-1}\right\|^{2}\right)}{2\left(1-\ell_{0}\left\|x_{m}-x_{0}\right\|\right)} \\
& \leq \frac{\ell_{0}\left(\left(s_{m}-t_{m}\right)^{2}+\left(t_{m}-t_{m-1}\right)^{2}\right)}{2\left(1-\ell_{0} t_{m}\right)}=s_{m}-t_{m}
\end{aligned}
$$

and

$$
\begin{aligned}
\left\|x_{m+1}-y_{m}\right\| & \leq \frac{\ell}{2} \frac{\left\|y_{m}-x_{m}\right\|^{2}}{1-\ell_{0}\left\|x_{m}-x_{0}\right\|} \\
& \leq \frac{\ell}{2} \frac{\left(s_{m}-t_{m}\right)^{2}}{1-\ell_{0} t_{m}}=t_{m+1}-s_{m},
\end{aligned}
$$

which complete the induction for (2.19), and 2.20).

We shall also show $x_{m+1} \in \bar{U}\left(x_{0}, r_{0}\right)$.

Indeed, we have as in 2.29 :

$$
\begin{aligned}
\left\|x_{m+1}-x_{0}\right\| & \leq\left\|x_{m+1}-y_{m}\right\|+\left\|y_{m}-x_{m}\right\|+\left\|x_{m}-x_{0}\right\| \\
& \leq t_{m+1}-s_{m}+s_{m}-t_{m}+t_{m}-t_{0}=t_{m+1} \leq r_{0} .
\end{aligned}
$$

It follows from Lemma 2.1 that sequences $\left\{x_{m}\right\},\left\{y_{m}\right\}$ are Cauchy in a Banach space $\mathcal{X}$, and as such they converge to their common limit $x^{\star} \in \bar{U}\left(x_{0}, r_{0}\right)$ (since $\bar{U}\left(x_{0}, r_{0}\right)$ is a closed set).

In view of the estimate

$$
\begin{aligned}
\left\|y_{m}-x_{m}\right\| & =\left\|\Gamma_{m} F^{\prime}\left(x_{0}\right) F^{\prime}\left(x_{0}\right)^{-1} F\left(x_{m}\right)\right\| \\
& \leq\left\|\Gamma_{m} F^{\prime}\left(x_{0}\right)\right\|\left\|F^{\prime}\left(x_{0}\right)^{-1} F\left(x_{m}\right)\right\| \leq s_{m}-t_{m},
\end{aligned}
$$

we conclude by letting $m \longrightarrow \infty$, that $F\left(x^{\star}\right)=0$.

Estimates 2.17), and (2.18) follow from 2.19), and 2.20), respectively by using standard majorization techniques [6], 7], [9].

The uniqueness part can be found in [2].

The part (b) of Theorem 2.2 follows from the estimate:

$$
\begin{aligned}
e_{m+1} & =x_{m+1}-x^{\star} \\
& =\frac{1}{6}\left((p-1) \Gamma_{m} F^{\prime \prime \prime}\left(x_{m}\right)+3\left(\Gamma_{m} F^{\prime \prime}\left(x_{m}\right)\right)^{2}\right) e_{m}^{3}+O\left(\left\|e_{m}\right\|^{4}\right),
\end{aligned}
$$

and has been obtained in [8, Theorem 4.1].

That completes the proof of Theorem 2.2. 
Remark 2.3. We compare our results with the ones obtained in [8].

Note that

$$
\ell_{0} \leq \ell
$$

holds in general, and $\frac{\ell}{\ell_{0}}$ can be arbitrarily large [2], [3], [6].

Case 1. Affine versus non-affine results. The results in [8] were obtained in non-affine invariant form. The advantages of affine invariant over nonaffine results have been explained in [6].

We shall compare our results with the ones in [8] by first turning their results into affine ones. This can simply be done by replacing operator $F$ by $\Gamma_{0} F$ in their proofs. We have estimate

$\left\|\Gamma_{0}\left(F^{\prime}(x)-F^{\prime}(y)\right)\right\| \leq\left\|\Gamma_{0}\right\|\left\|F^{\prime}(x)-F^{\prime}(y)\right\| \leq\left\|\Gamma_{0}\right\| \bar{\ell}\|x-y\|$,

and

$$
\ell \leq\left\|\Gamma_{0}\right\| \bar{\ell}
$$

where,

$$
\left\|F^{\prime}(x)-F^{\prime}(y)\right\| \leq \bar{\ell}\|x-y\| .
$$

Case 2. $\ell=\ell_{0}$. Conditions (2.9)-2.13) together with

$$
q=\ell \eta<.32666
$$

were essentially used in [8]. In this case, condition (2.38) implies 2.5), and our results coincide with the ones in [8].

Case 3. $\ell \neq \ell_{0}$. Denote by $\left\{\bar{t}_{n}\right\},\left\{\bar{s}_{n}\right\}$ majorizing sequences obtained as $\left\{t_{n}\right\},\left\{s_{n}\right\}$, respectively by simply replacing $\ell_{0}$ by $\ell$ in $(2.1)-2.4$. These sequences were essentially used in [8]. However, under 2.38, an inductive argument shows:

$t_{n}<\bar{t}_{n}, \quad s_{n}<\bar{s}_{n}, \quad t_{n+1}-s_{n}<\bar{t}_{n+1}-\bar{s}_{n}, \quad s_{n}-t_{n}<\bar{s}_{n}-\bar{t}_{n}$,

and

$$
r_{0}<r^{0}
$$

where,

$$
r^{0}=\lim _{n \longrightarrow \infty} \bar{t}_{n}
$$

Hence, the radius of convergence, error bounds on the distances $\|$ $x_{n+1}-y_{n}\|,\| y_{n}-x_{n}\|,\| x_{n}-x^{\star}\|,\| y_{n}-x^{\star} \|,(n \geq 0)$ are improved, under the same or weaker hypotheses and computational cost. That is, the applicability of (NCTM) has been expanded. 
Case 4. The advantages obtained above extend to (MNCTM):

$$
\begin{aligned}
& x_{0} \in \mathcal{D}, \\
& x_{n}=x_{n-1}-\Gamma_{n-1} F\left(x_{n-1}\right), \quad n=1,2, \cdots, N_{0}, \\
& \bar{x}_{0}=x_{N_{0}}, \\
& \bar{y}_{k-1}=\bar{x}_{k-1}-F^{\prime}\left(\bar{x}_{k-1}\right)^{-1} F\left(\bar{x}_{k-1}\right), \\
& \bar{z}_{k-1}=\bar{x}_{k-1}+p\left(\bar{y}_{k-1}-\bar{x}_{k-1}\right), \quad p \in(0,1], \\
& \bar{x}_{k}=\bar{x}_{k-1}-\frac{1}{p^{2}} F^{\prime}\left(\bar{x}_{k-1}\right)^{-1}\left(\left(p^{2}+p-1\right) F\left(\bar{x}_{k-1}\right)+F\left(\bar{z}_{k-1}\right)\right),
\end{aligned}
$$

where, $N_{0}$ is a uniquely determined integer [8].

We leave the details of this case to the motivated reader.

Similar work, where linearly convergent method

$$
x_{n+1}=x_{n}-\Gamma_{0} F\left(x_{n}\right)
$$

is used instead of quadratically convergent method, or modified Newton-like method (MNLM)

instead of (NLM)

$$
x_{n+1}=x_{n}-A\left(x_{0}\right)^{-1} F\left(x_{n}\right)
$$

$$
x_{n+1}=x_{n}-A\left(x_{n}\right)^{-1} F\left(x_{n}\right)
$$

in connection with (MNCTM) has been carried in 4], and 5], respectively.

\section{CONCLUSION}

In order to approximate a locally unique solution of a nonlinear equation in a Banach space, we provided a semilocal convergence analysis for (NCTM), involving a Fréchet differentiable operator.

We provided a semilocal convergence analysis with the following advantages over the work in [8]: larger convergence domain, weaker sufficient convergence conditions, and tighter error bounds. Note that these advantages are obtained under the same or weaker computational cost as in [8].

\section{REFERENCES}

1. S. Amat, S. Busquier, J.M. Gutiérrez, Geometric constructions of iterative functions to solve nonlinear equations, J. Comput. Appl. Math. 157 (2003), 197-205. 1

2. I.K. Argyros, On the Newton-Kantorovich hypothesis for solving equations, J. Comput. Appl. Math. 169 (2004), 315-332. 2, 2.3 
3. I.K. Argyros, A unifying local-semilocal convergence analysis and applications for twopoint Newton-like methods in Banach space, J. Math. Anal. Appl. 298 (2004), 374-397. 2. 2.3

4. I.K. Argyros, Approximating solutions of equations using Newton's method with a modified Newton's method iterate as a starting point, Revue d'Analyse Numér. Th. Approx. 36 (2007), 123-137. 2.3

5. I.K. Argyros, Approximating solutions of equations by combining Newton-like methods, J. Korea Soc. Math. Educ. Ser. B Pure Appl. Math. 15 (2008), 35-45. 2.3

6. I.K. Argyros, Convergence and Applications of Newton-type iterations, Springer-Verlag Publ., New York, 2008. 2, 2, 2, 2.3

7. I.K. Argyros, F. Szidarovsky, The theory and applications of iteration methods, Systems Engineering Series, CRC Press, Boca Raton, Florida, 1993. 2, 2

8. J.A. Ezquerro, M.A. Hernández, An optimization of Chebyshev's method, J. Complexity, in press. (document), 1, 2, 2.3, $2.3,2.3,2.3,2$

9. L.V. Kantorovich, G.P. Akilov, Functional analysis in normed spaces, Pergamon Press, Oxford, 1982.2, 2

10. S. Weerakoon, T.G.I. Fernando, A variant of Newton's method with accelerated third-order convergence, Appl. Math. Lett. 13 (2000), 87-93. 1

${ }^{1}$ Cameron university, Department of Mathematics Sciences, Lawton, OK 73505, USA.

E-mail address: iargyros@cameron.edu

2 Poitiers university, Laboratoire de Mathématiques et Applications, Bd. Pierre et Marie Curie, Téléport 2, B.P. 30179, 86962 Futuroscope Chasseneuil Cedex, France.

E-mail address: said.hilout@math.univ-poitiers.fr 